\title{
JOINS OF EUCLIDEAN ORBITAL TOPOLOGIES
}

\author{
ELLEN CLAY \\ St. John Fisher College \\ 3690 East Avenue \\ Rochester, NY 14618
}

(Received March 20, 1995 and in revised form August 25,1995)

\begin{abstract}
This paper is concerned with joins of orbital topologies especially on the orbit of the reals with the usual topology.
\end{abstract}

KEY WORDS AMD PHRASES. Orbital topologies, joins, connected, locally compact. 1995 AMS SUBJECT CLASSIFICATION CODES. 54

The importance of comparing two different topologies on the same set was noted by Garrett Birkhoff in 1936 [1]. Let $\mathrm{X}$ be a set and $\mathrm{L}(\mathrm{X})$ be the lattice of all topologies on $\mathrm{X}$. If $\mathrm{f}$ is a bijective function from $X$ to $X$ and $\tau$ is a fixed topology on $X$, then we can define $\tau_{f}=\{f(U) \mid U \in \tau\}$. Note that $\tau_{f}$ is a topology. Let $\mathscr{I}$ be the set of all bijections from $X$ to $X$. Define $\left\{\tau_{f} \mid f \in \mathscr{I}\right\}$ to be the orbit of $\tau$ in $L(X)$. The topologies in this orbit are homeomorphic to each other. Also note that for all bijective functions $f$ and $g$, there exists a bijection $h$ such that $\tau_{f} \vee \tau_{g}{ }^{\prime}$ is homeomorphic to $\tau \vee \tau_{h}$

Throughout this paper we will refer to the orbit of the usual topology on the reals as the Euclidean Orbit. All functions will be bijective, and $\{(x, f(x)) \mid x \in X\}$, the graph of $f$, will be denoted $G(f)$.

Bourbaki [2] showed $\left(X, \tau \vee \tau_{f}\right)$ is homeomorphic to $\{(x, x) \mid x \in X\}$ with the relative topology of $\tau \times \tau_{f}$ via $h(x)=(x, x)$. Clearly, $\left(X \times X, \tau \times \tau_{f}\right)$ is homeomorphic to $(X \times X, \tau \times \tau)$ via $F(x, y)=\left(x, f^{-1}(y)\right)$. Hence $\left(X, \tau \vee \tau_{f}\right)$ is homeomorphic to $\left(G\left(f^{-1}\right), \tau x \tau\right)$. It is this graph which will help us discover properties of $\left(X, \tau \vee \tau_{\mathfrak{f}}\right)$.

Note that if $\mathrm{X}$ is a metric space, it is trivial to see that $\tau \vee \tau_{\mathrm{f}}$ is metric. But locally compact is not so clear. Given a locally compact Hausdorff space, we have the following: 
THEOREM 1. Let $G^{\#}(f)=\operatorname{cl}(G(f))-G(f) . \tau \vee \tau_{f-1}$ is locally compact if and only if $\operatorname{cl}\left(G^{\#}(f)\right) \cap G(f)=\varnothing$

PROOF. If $\operatorname{cl}\left(G^{*}(f)\right) \cap G(f) \neq \varnothing$, then let $p \in \operatorname{cl}\left(G^{*}(f)\right) \cap G(f)$. Then $p \notin G^{\#}(f)$; hence $p$ is in the derived set. Let $C$ be a compact neighborhood of $p$ in $G(f)$; then there exists an open $V \subset X^{2}$ such that $V \cap G(f) \subset C$ and $V \cap G(f)$ is compact. Since $c l(V)$ is a neighborhood of $p$ in $X^{2}$, there exists a point $q \in V$ such that $q \in G^{*}(f)$. Let $\left\{V_{\alpha}\right\}$ be a basis at $q$. Since $X$ is regular, we can assume there is a basis element $\mathrm{V}_{\beta}$ such that $\operatorname{cl}\left(\mathrm{V}_{\beta}\right) \subseteq \mathrm{V}_{\alpha}$ Let $\mathrm{U}_{\alpha}=\mathrm{X}-\operatorname{cl}\left(\mathrm{V}_{\beta}\right)$; then $\left\{\mathrm{U}_{\alpha}\right\}$ covers $\mathrm{X}$ - q. Hence $\left\{\mathrm{U}_{\alpha}\right\}$ covers $\mathrm{G}(\mathrm{f}) \cap \mathrm{cl}(\mathrm{V})$. But since $\mathrm{G}(\mathrm{f}) \cap \mathrm{cl}(\mathrm{V})$ is compact, there exists a finite subcover $\left\{U_{\alpha_{1}}, \ldots, U_{\alpha_{m}}\right\}$ which covers $G(f) \cap \mathrm{cl}(V)$. Let $U$ be the union of the subcover. Then $U$ covers $\mathrm{G}(\mathrm{f}) \cap \mathrm{cl}(\mathrm{V})$. This is a contradiction since $\mathrm{q} \in \mathrm{cl}(\mathrm{G}(\mathrm{f}))$, but $\mathrm{q} \notin \mathrm{U}$.

Now suppose $\operatorname{cl}\left(G^{*}(f)\right) \cap G(f)=\varnothing$ and let $p \in G(f)$. Then there is an open $U$ containing $p$ such that $U \cap G^{*}(f)=\varnothing$. Also we can find an open neighborhood $V$ of $p$ such that $\mathrm{cl}(\mathrm{V}) \subset \mathrm{U}$. Since $\mathrm{cl}(\mathrm{V}) \cap \mathrm{cl}\left(\mathrm{G}^{\prime \prime}(\mathrm{f})\right)=\varnothing, \mathrm{G}(\mathrm{f}) \cap \mathrm{cl}(\mathrm{V})$ is closed. Therefore, $\mathrm{G}(\mathrm{f})$ is locally compact.

For the remainder of this paper, we restrict ourselves to the Euclidean orbit. In the Euclidean orbit we know that $\tau=\tau_{f}$ only if $f$ is continuous and that since $\tau$ is connected, $\tau_{f}$ is also, but what about $\tau \vee \tau_{\mathfrak{q}}$ ?

THEOREM 2. $\tau \vee \tau_{f}$ is connected if and only if $\tau=\tau_{f}$.

PROOF. If $\tau=\tau_{\mathrm{f}}$, then $\tau \vee \tau_{\mathrm{f}}=\tau$, hence it is connected. Now, if $\tau \neq \tau_{\mathrm{f}}$, then $\mathrm{f}$ is not continuous. But $f$ is bijective so neither is the inverse of $f$. Let $x_{0}$ be a point of discontinuity of $f^{-1}$. Then there is a sequence $\left\{x_{n}\right\}$ such that $\left\{x_{n}\right\} \rightarrow x_{0}$, but $\left\{f^{-1}\left(x_{n}\right)\right\} \nrightarrow f^{-1}\left(x_{0}\right)$. Suppose $\left\{f^{-1}\left(x_{n}\right)\right\}$ is bounded. Then there exists a convergent subsequence $\left\{f^{-1}\left(x_{n_{k}}\right)\right\}$. Let $\lim \left\{f^{-1}\left(x_{n_{k}}\right)\right\}=y$. Without loss of generality, let $y>f^{-1}\left(x_{0}\right)$. Then there is an $M>0$ such that for every $n_{k}>M, f^{-1}\left(x_{n_{k}}\right)>f^{-1}\left(x_{0}\right)$. Let $n_{j}>M$ then $f^{-1}\left(x_{n_{j}}\right)>f^{-1}\left(x_{0}\right)$. Now consider the vertical ray $A=\left\{(a, b) \mid a=x_{0}\right.$ and $b>f^{-1}\left(x_{n}\right)$ and let $x_{n} \in R$ such that $\left|f^{-1}\left(x_{n}\right)-y\right| \leq\left|f^{-1}\left(x_{n}\right)-y\right|$ and without loss of generality, let $x_{0}<x_{n}$. Consider the horizontal line segment $B=\left\{(a, b) \mid x_{0} \leq a<x_{n_{a}}\right.$ and $\left.b=f^{-1}\left(x_{n},\right)\right\}$. Also, consider the vertical ray $C=\left\{(a, b) \mid a=x_{n}\right.$, and $\left.b \leq f^{-1}\left(x_{n}\right)\right\}$.

Since $f^{-1}$ is an injective function, $(A \cup B \cup C) \cap G\left(f^{-1}\right)=\varnothing$. Now 
$\left(x_{n}, f^{-1}\left(x_{n}\right)\right)$ and $\left(x_{0}, f^{-1}\left(x_{0}\right)\right)$ lie in separate components of $\mathbf{R}-(A \cup B \cup C)$. So in the bounded case, $\tau \vee \tau_{\mathrm{f}}$ is not connected. The unbounded case is similar.

COROLLARY 3. $\tau \vee \tau_{\mathrm{f}}$ is path-connected if and only if it is connected.

THEOREM 4. Let $D(f)=\{x \mid f$ is discontinuous at $x\}$. If $D\left(f^{-1}\right)$ is a discrete subset of $\mathbf{R}$, then $\tau \vee \tau_{\mathrm{f}}$ is locally connected.

The proof is very similar to that of Theorem 2 and hence is omitted.

COROLLARY 5. $\tau \vee \tau_{\mathrm{f}}$ is locally path connected if and only if $\tau \vee \tau_{\mathrm{f}}$ is locally connected.

THEOREM 6. If $\tau \vee \tau_{\mathrm{f}}$ is locally connected, then $\tau \vee \tau_{\mathrm{f}}$ is locally compact.

PROOF. Since $\tau \vee \tau_{f}$ is locally connected, each component $C$ of $\left(G\left(f^{-1}\right), \tau \vee \tau\right)$ is open. Now $\pi_{1}(C)$ and $\pi_{2}(C)$ are connected subsets of the reals, therefore intervals. Now $f^{-1} \mid \pi_{1}(C)$ múst be monotone, otherwise we would have points $a, b, c \in \pi_{1}(C)$ with $a<b<c$ such that $f^{-1}(a) \in \pi_{2}(C)$ and without loss of generality $f^{-1}(b)>f^{-1}(a)$. Now suppose $f^{-1}(c)<f^{-1}(b)$. If $\mathrm{f}^{-1}(\mathrm{c})>\mathrm{f}^{-1}(\mathrm{a})$, then the set $\left\{(\mathrm{a}, \mathrm{y}) \mid \mathrm{y} \geq \mathrm{f}^{-1}(\mathrm{c})\right\} \cup\left\{\left(\mathrm{x}, \mathrm{f}^{-1}(\mathrm{c})\right) \mid \mathrm{a} \leq \mathrm{x} \leq \mathrm{b}\right\} \cup\left\{(\mathrm{b}, \mathrm{y}) \mid \mathrm{y} \leq \mathrm{f}^{-1}(\mathrm{c})\right\}$ disconnects C. If $\mathrm{f}^{-1}(\mathrm{c})<\mathrm{f}^{-1}(\mathrm{a})$, then the set $\left\{(\mathrm{c}, \mathrm{y}) \mid \mathrm{y} \geq \mathrm{f}^{-1}(\mathrm{a})\right\} \cup\left\{\left(\mathrm{x}, \mathrm{f}^{-1}(\mathrm{a})\right) \mid \mathrm{b} \leq \mathrm{x} \leq \mathrm{c}\right\} \cup$ $\left\{(b, y) \mid y \leq f^{-1}(a)\right\}$ disconnects $C$. This shows that a function which increases from a to $b$ must continue to increase, the decreasing case is similar. So we have $f^{-1} \mid \pi_{1}(C)$ is a monotonic function from $\pi_{1}(C)$ to $\pi_{2}(C)$, hence $f^{-1}$ is continuous on $\pi_{1}(C)$. Therefore $G\left(f^{-1} \mid \pi_{1}(C)\right)$ is homeomorphic to an interval, thus locally compact. Hence $\tau \vee \tau_{\S}$ is locally compact.

The converse of this theorem is not, however, true. The following counter example illustrates this.

$$
\begin{array}{lll}
1 & x=-1 \\
-1 & x=0 \\
x-1 & \frac{1}{x} \in Z^{+} \\
& x+1 \quad & \frac{1}{x} \in\{Z-\{-1\}\} \\
& x+1 & x \in(-1,0) \text { and } \frac{1}{x+1} \in Z^{+} \\
& x-1 & x \in(0,1) \text { and } \frac{1}{x-1} \in Z^{-} \\
& \text {Otherwise }
\end{array}
$$


E. CLAY

The graph of $f$ is locally compact, but there is no connected neighborhood about $(0,-1)$.

\section{REFERENCES}

[1] Birkhoff, Garrett, "On the combination of topologies," Fund. Math. 26 (1936), 156-166.

[2] Bourbaki, Nicolas, General Topology Part I (Addison - Wesley Publishing Company, Massachusetts, 1966). 


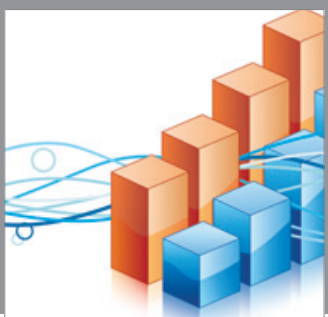

Advances in

Operations Research

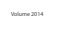

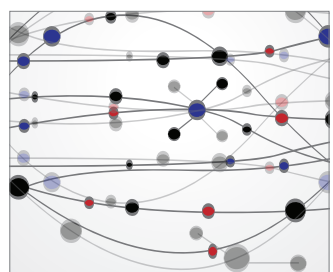

\section{The Scientific} World Journal
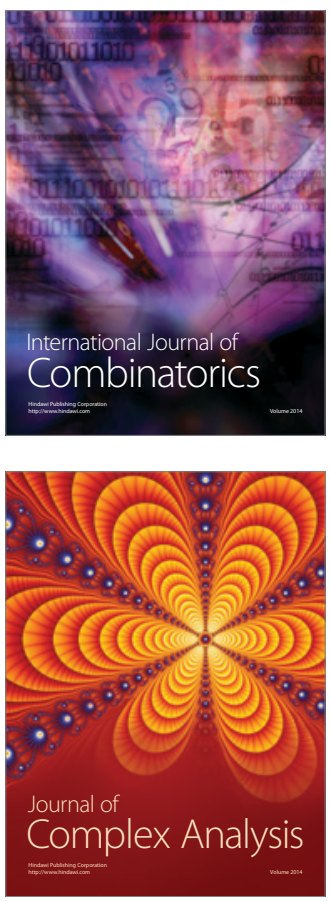

International Journal of

Mathematics and

Mathematical

Sciences
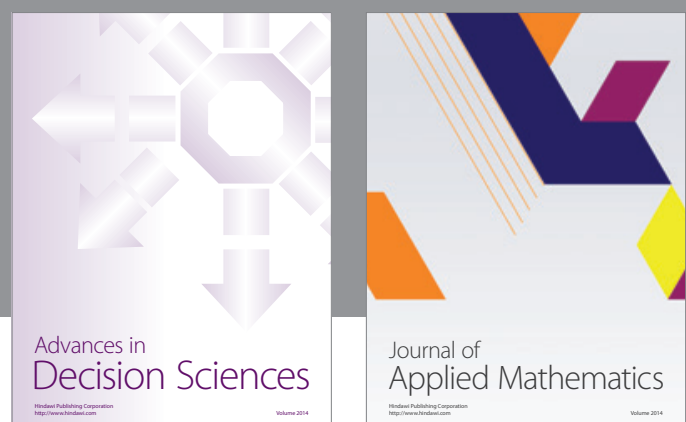

Journal of

Applied Mathematics
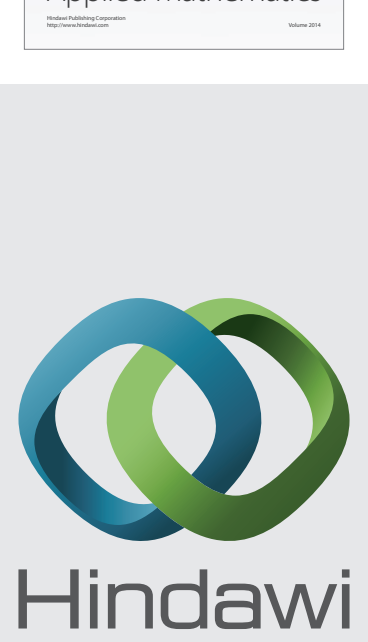

Submit your manuscripts at http://www.hindawi.com
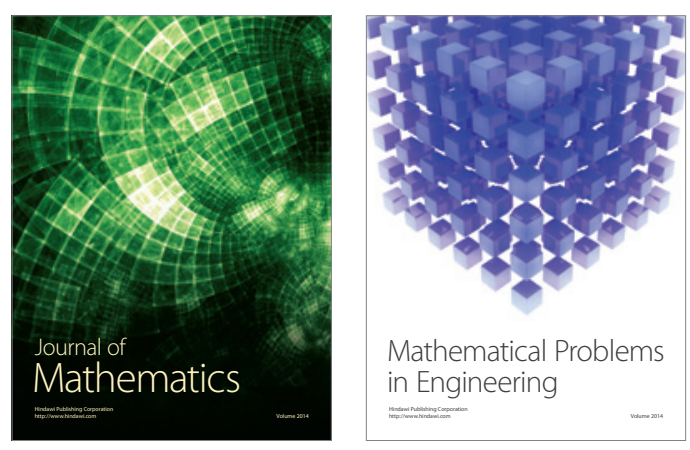

Mathematical Problems in Engineering
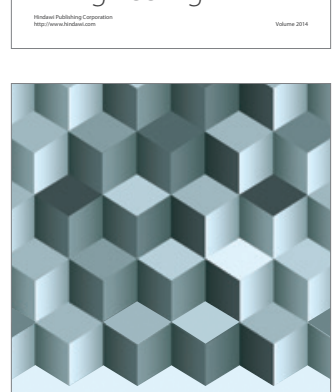

Journal of

Function Spaces
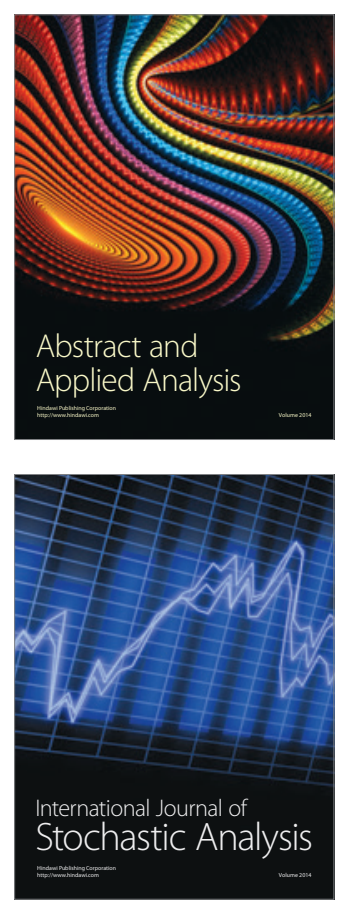

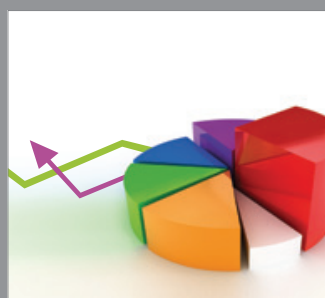

ournal of

Probability and Statistics

Promensencen
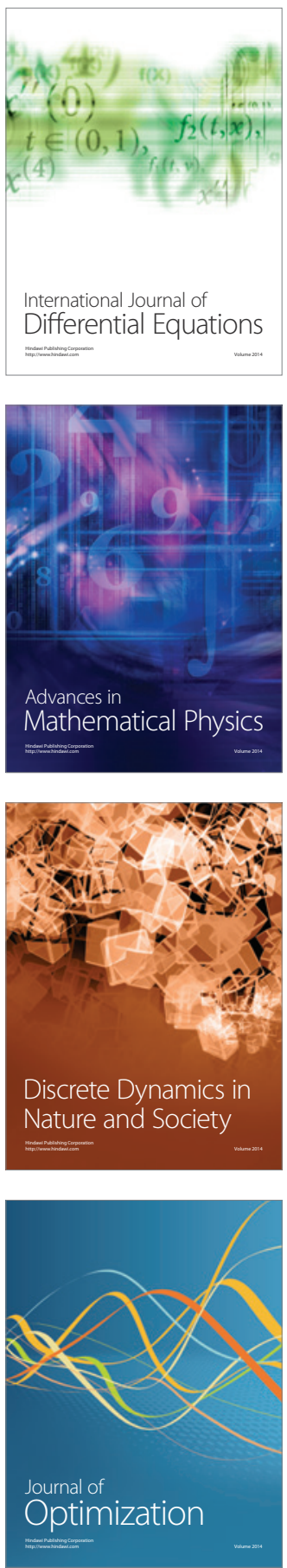